\title{
BMJ Open Challenges and opportunities for involving patients and the public in acute antimicrobial medicine development research: an interview study
}

Andy Gibson, ${ }^{1}$ Michele Kok, ${ }^{1}$ David Evans, ${ }^{1}$ Sally Grier, ${ }^{2}$ Alasdair MacGowan ${ }^{2}$

To cite: Gibson A, Kok M, Evans D, et al. Challenges and opportunities for involving patients and the public in acute antimicrobial medicine development research: an interview study. BMJ Open 2019;9:e024918. doi:10.1136/ bmjopen-2018-024918

- Prepublication history for this paper is available online. To view these files, please visit the journal online (http://dx.doi org/10.1136/bmjopen-2018024918).

Received 20 June 2018 Revised 16 0ctober 2018 Accepted 17 October 2018
Check for updates

(c) Author(s) (or their employer(s)) 2019. Re-use permitted under CC BY-NC. No commercial re-use. See rights and permissions. Published by BMJ.

${ }^{1}$ Department of Health and Social Sciences, University of West England, Bristol, UK ${ }^{2}$ Department of Infection Sciences, Southmead Hospital, North Bristol NHS Trust, Bristol, UK

Correspondence to Dr Andy Gibson andy.gibson@uwe.ac.uk

\section{ABSTRACT}

Objectives To explore what approaches to patient and public involvement (PPI) in antimicrobial medicines development are currently being used, what the impacts of PPI are on antimicrobial medicines development and what the barriers are to its implementation.

Design Interview study.

Setting Antimicrobial medicines development research. Participants Principal investigators known to have led studies involving PPI or expressed an interest in PPI. Results There is very little published work on PPI in antimicrobial research. Individual interviewees expressed scepticism about the contribution that PPI could make to different stages of the medicines development life cycle but collectively identified a range of potential benefits of PPI covering most stages of the medicines development process.

Conclusions A major issue in developing PPI in antimicrobial medicines development research will be in overcoming the view that, at best, PPI has only a marginal contribution to make in this area of research. The findings from this study, although mixed, suggest that well-designed PPI has an untapped potential to enhance antimicrobial research.

\section{BACKGROUND: PATIENT AND PUBLIC INVOLVEMENT IN HEALTH RESEARCH}

There is a rapidly growing interest in patient and public involvement (PPI) in health research. INVOLVE, a UK-based advisory group on PPI funded by the National Institute for Health Research, defines involvement as research being carried out 'with' or 'by' members of the public rather than 'to', 'about' or 'for' them. ${ }^{1}$ This is distinct from either disseminating information about research to the public or people participating as subjects of the research. Examples include acting as joint grant holders or co-applicants on a research project, involvement in identifying research priorities, participating as members of a project advisory or steering group, commenting on and developing patient information leaflets or other research materials and users and/or carers themselves carrying out research.

Within the literature, there are ongoing discussions about what 'good' PPI looks like. In recent years, the term 'co-production' has gained prominence. These discussions reflect longer term concerns regarding the impact of entrenched power asymmetries between researchers and the public on the conduct and practice of involvement. ${ }^{2-4}$

PPI is advocated on several groundsit helps to ensure that health research is conducted ethically, it improves the quality of research design and it helps in the production of research findings that address patient and public concerns. Underlying these claims is the assertion that PPI provides access to an additional source of knowledge, that is, experiential knowledge, which is different from, but equally as important as, scientific or professional knowledge, in carrying out health research. ${ }^{2}$

PPI is an international movement, with comparable initiatives in other countries. In the USA, the Patient Centered Outcomes Research Institute is a major source of research funding, focused on question generation, patient-centred clinical effectiveness research and broad dissemination. The Strategy for Patient Orientated Research in Canada and the Consumer and Community Health Research Forum (Involving People in Research) in Australia include consumer-based research and a strong consumer knowledge base. There are also more targeted interventions such as the European Patients' Academy on Therapeutic Innovation (EUPATI) which aims to increase the number of patients who are knowledgeable of the medicines development process and 
therefore able to act as effective advocates and advisors in medicines research.

Evidence of PPI in development and research plans is now a requirement for many UK-based medical research funding bodies. In Europe, the Innovative Medicines Initiative places a strong emphasis on the importance of PPI in health research.

There has been much debate about the correct terminology to use when referring to members of the public who are involved in designing and carrying out research. In this paper, we use the term 'public contributors' to cover people who may have had direct experience of an infection, their carers and members of the public who may have a more general interest in antimicrobial research.

\section{PPI IN ACUTE ANTIMICROBIAL MEDICINES RESEARCH}

Despite the trend towards increasing PPI in research, there has been apparently relatively little interest in public involvement in antimicrobial research. Several authors of this paper (DE, AG, SG, AM) were involved in carrying out a systematic review to identify the extent, quality and impact of PPI in antimicrobial drug development research. ${ }^{5}$ No relevant studies were found, apart from one protocol paper with a brief mention of PPI. Given the rapidly growing international problem of antimicrobial resistance, this is an important area of research and public concern in terms of both the need to develop new antimicrobials and the stewardship of existing antimicrobials. ${ }^{6}$

There may be a number of reasons why public involvement is not prominent in antimicrobial research. One is that researchers involved in antimicrobial research may be unaware of the potential benefits of PPI. There is a growing evidence base for the positive impacts of PPI on research. This includes impacts on setting the research agenda, intervention development, choosing outcome measures, data collection, analysis of data and writing up and dissemination. ${ }^{78}$ However, there is little consensus on the aims, methodology and appropriate outcome measures for evaluating PPI. This partly reflects the different requirements of funders, researchers and public contributors in developing evidence of the benefits of PPI, which in turn makes it difficult to draw firm conclusions.

Another barrier might be the biomedical nature of much of the research. It may be felt that there is less scope for PPI to beneficially impact on laboratory-based research, as opposed to more applied health services research. That being said, there are well-documented cases of public involvement in other types of medicines development research, perhaps the most notable being Epstein's work on the development of the relationship and interaction between AIDS activists and AIDS research. ${ }^{9}$

The example of AIDS activism is helpful but the focus in this paper is on acute rather than chronic infections, which leads us to one final, and perhaps, crucial issue, the temporary nature of most microbial infections. With many long-term conditions, there are well-established patient groups that have advocated for the rights of their members to be heard in decisions about service provision and research that affects them. The AIDS activism mentioned above is one example, but groups representing people with mental health problems, physical disabilities and chronic conditions such as diabetes also spring to mind. The existence of these groups and networks make it easier for researchers to contact appropriate patients and carers and involve them in their work. In contrast, there are few, if any, groups, representing people who have experienced acute microbial infections. There are groups for conditions which may make people prone to recurrent infections, for example, bronchiectasis, chronic obstructive pulmonary disease and cystic fibrosis. These are important groups to be aware of but it should be borne in mind that people who suffer recurrent chronic infections due to pre-existing diseases may have a different experience and knowledge of their disease compared with those who experience a single and unexpected severe acute infection.

The long-term nature of some conditions also makes it possible for researchers to build more sustained relationships with these patients across the lifetime of a project, leading to more substantial involvement. Patients often experience acute bacterial infection as a one-off experience which is either successfully treated with antibiotics or may be fatal. Thus, involving patients in research on treating certain types of infections may be more problematic. What was unknown at the beginning of this study was the extent to which researchers were able to overcome these barriers and successfully involve patients in antimicrobial medicines development research.

The authors of this paper are part of a larger European programme of research to develop new antimicrobial agents (COMBACTE-MAGNET). Combating bacterial resistance in Europe-molecules against Gram-negative infections (www.combacte.com): A consortium seeking new ways of treating multi-resistant bacterial infections. The authors have the responsibility to encourage the development of PPI within the COMBACTE-MAGNET programme and are based in Work Package 6i. It is therefore important to identify any relevant work on PPI in antimicrobial research that could be built on for the programme.

Our aims were therefore to identify any relevant PPI work taking place in antimicrobial research within the UK or elsewhere within the COMBACTE-MAGNET programme and to collect data on the approaches to PPI used and the impact of PPI.

\section{RESEARCH QUESTIONS}

1. What approaches to PPI in antimicrobial medicines development are currently being used? 
2. What are the impacts of PPI on antimicrobial medicines development and what are the barriers to its implementation?

\section{RESEARCH DESIGN}

The majority of the data were collected by means of telephone interviews. Telephone interviews were chosen because the potential participants were geographically dispersed, based in the UK, the USA, Vietnam and mainland Europe. The numbers of people we were able to identify carrying out PPI in antimicrobial research were also relatively small. It was therefore possible to interview all our potential participants. Telephone interviews also offered a practical way to develop a more detailed understanding of the process and outcomes of PPI in antimicrobial research than would be possible using other methods such as a questionnaire.

\section{Population and sample}

The population was researchers involved in antimicrobial research within the UK and the COMBACTE-MAGNET programme. Potential interviewees were identified through our contacts within the COMBACTE-MAGNET programme. We also contacted INVOLVE to identify potential contacts but without any success. We had hoped that a rapid review of the literature in this area of work would yield some contacts. ${ }^{5}$

We had originally planned to carry out a purposive sample of identified contacts, but because we were only able to identify a small number of people to participate, all identified contacts were interviewed. The people identified were all known to our team. AM is an expert in this area of research and was particularly helpful in identifying contacts and providing introductions. This was significant given the lack of published literature to follow up or contacts from other sources. We interviewed nine people in total-all were male principal investigators with established research track records, five were based in the UK, one was based in Vietnam but the interview related to work carried out in the UK, one based in the USA, one in Switzerland and one in the Netherlands. All of the researchers who had carried out work in the UK had experience of PPI in their research projects. It transpired that the three non-UK interviewees did not have experience of carrying out PPI but did have opinions on the potential benefits of PPI. We have included their comments in this study because they illustrate some of the barriers to developing PPI in antimicrobial research.

\section{Data collection}

Interviews were conducted using a semi-structured approach. ${ }^{10}$ This approach allowed us to ensure that important topics were covered while allowing the flexibility for the interviewees to raise any issues they may have wished to. The interviews were conducted by $\mathrm{AG}$ and $\mathrm{MK}$ and on average lasted $20 \mathrm{~min}$. The topic guide was structured to obtain the following information:

- Skills or previous background in PPI
- Perceived value of PPI in antimicrobial medicines development research

- Where in the medicines development process PPI is carried out

- Recruitment and maintenance of PPI groups

- Methods of involvement

The topic guide was developed by the authors and was informed by our wider discussion with members of the Patient and Public Involvement Panel for Antimicrobial Drugs (PPIPAD) at regular bimonthly meetings.

In addition to the areas listed above, issues specific to a particular research project were pursued during the interviews, where they were relevant to the aims of this paper.

\section{Patient involvement}

This paper is part of a larger project on public involvement in antimicrobial medicines development which includes the development of a toolkit for PPI in antimicrobial medicines development research. The work of the project is guided by members of PPIPAD. Members of the panel confirmed that our research questions were important issues to investigate. Discussions with PPIPAD and the project team informed the development of the interview schedule. PPIPAD members were not involved in the recruitment to this study. We will discuss with PPIPAD the potential for further dissemination of the findings from this work.

\section{DATA ANALYSIS}

The data were analysed by AG. The approach to data analysis was guided by the work of Ritchie and Spencer. ${ }^{11}$ This approach was taken because it was designed with research related to policy issues in mind and because it allows for themes to be developed both inductively and deductively from the data, that is, it was possible to explore topics that arose from our original research questions while remaining open to identifying issues and concerns related to PPI as identified by our interviewees.

The results are presented following the themes developed by the analysis process as follows:

- Responsibility for carrying out PPI

- Basis for public involvement

- Time and resource implications

- Recruitment of public contributors

- PPI activities undertaken

- Value added

- Main barriers to public involvement

\section{RESULTS}

\section{Responsibility for carrying out PPI}

As stated above, three of our interviewees were interested in the potential contribution of PPI but had no experience of doing PPI. The other five interviewees were responsible for ensuring that PPI work was carried out in their projects in line with any commitments made in their original funding applications. However, in practice, responsibility 
for PPI was usually delegated to a specific member of the team who was accountable for the day-to-day running of PPI activities.

\section{Basis for public involvement}

It is common in the literature on involvement to argue that public contributors possess important 'lived experience' of a particular condition which needs to be considered, alongside other forms of knowledge, for example, professional and scientific, when designing research. ${ }^{12}$ However, in antimicrobial research, we are dealing with acute infections which people may not have experienced before. This, combined with the laboratory-based nature of antimicrobial research, led to a questioning of the value of PPI by some of our interviewees. As one of our study participants put it, 'I don't think patients have any major role to play, honestly.' (interview 1)

However, other interviewees did not feel that this lack of 'lived experience' of a condition disqualified public contributors from being able to add value to antimicrobial research. The ability to provide an 'alternative perspective' was seen as important. One of our interviewees, for example, talked about his experience of involving young people in the running of trials related to vaccination programmes. Not only did they gain valuable information about the best time and place to contact potential participants, the young people involved also acted as 'research ambassadors', explaining the relevance and importance of participating in research to other young people and helping to create a 'research engaged community'. (interview 3 )

Furthermore, this interviewee felt that involvement of this kind

'Empowers researchers to know that they are taking the views of our research subjects into account in terms of the importance of our research and the way that we do it.'

It was notable that the interviewees with some direct experience of PPI were generally more positive about the potential of PPI to aid their research than those with none.

\section{Time and resources}

The need to allocate adequate time and resources for PPI was noted by interviewees. At a minimum, a budget is required to pay for the expenses of public contributors. It was also acknowledged that building relationships with a group of public contributors takes time but, as one interviewee noted, 'Much less time or trouble than working with clinical contributors.' (interview 2)

The need for time and resources was not necessarily seen as a problem, particularly if justified by clear benefits from PPI. However, one interviewee did raise concerns about PPI adding, 'an additional layer of bureaucratic complications.' (interview 1)

None of the interviewees provided formal training and support on involvement to their public contributors, although most provided informal support, for example, by explaining a particular research project and the planned role of public contributors.

Nevertheless, one interviewee was sceptical of the value that public contributors could add without significant training and support because of the complexity of the issues raised by antimicrobial resistance and the development of new medicines to combat this. He felt that what was needed was

'.. a well-educated elite representing patient groups who understand what we are talking about' (interview 8)

His concern was that this would be difficult to achieve given the short-term nature of acute infections.

\section{Recruitment of public contributors}

Recruitment was seen as a major problem by several interviewees because of the acute nature of most infections and the lack of easily identifiable patient groups to work with. It should be noted that this is not a problem unique to research in acute infections. Other forms of interaction with healthcare, for example, emergency care, are similarly episodic.

In most examples where PPI had taken place, contributors were recruited via pre-existing involvement networks and contacts. As one interviewee puts it, we 'beg, borrow or steal' (interview 4). Only one of the interviewees had set up a public advisory group specifically for antimicrobial research projects-this was a relatively recent innovation and was seen as a way of dealing with recruitment difficulties (interview 4).

Furthermore, several interviewees said that they regularly worked with an informal group of public contributors across different projects. One interviewee said that they drew on a group of approximately 10 people to work with on various projects since 2010 (interview 2).

It can clearly be beneficial and time efficient to work with public contributors with whom the researchers had already established a working relationship. This may also be a way of coping with the difficulty of engaging with this group of patients as noted earlier in this paper. One interviewee also commented about the potential difficulties caused by people dropping out of activities due to illness (interview 2). Having a core group to work with may help to minimise the impact of this kind of problem.

However, there were some concerns raised about how 'representative' public contributors were. One interviewee talked about most public contributors being, 'White, middle class types' (interview 1) and another commented on the problem of bias, that is, that public contributors may have personal interests which they may wish to pursue through their involvement in research (interview 2).

One interviewee (interviewee 7) described the very valuable contribution made by one public contributor but was concerned that this person had a very specific interest and motivation to become involved in the research which 
was not representative of the general population. This was particularly pronounced since other public contributors dropped out during the lifetime of the project leaving this person as the sole public contributor.

\section{PPI activities}

Despite the scepticism expressed by some as to the value of public involvement in antimicrobial research, interviewees described a wide range of activities that public contributors had undertaken in their research. These included, advising on confidentiality issues related to bioinformatics, guideline development for the use of antimicrobials, research agenda setting, preparing ethics applications, reviewing interview schedules, writing lay summaries, selecting outcome measures and involvement in planning and running trials. Many of these examples resonate with reports on the role that PPI plays in other forms of research. ${ }^{78}$

However, there were some potential areas of PPI work in antimicrobial research that are not reflected in this broader literature. One interviewee talked about the importance of PPI in making judgements about the 'trade-off between toxicity and efficacy' (interview 8 ) and another talked about the importance of working with patients and carers to design dosage regimes.

Another potential area for PPI to make a contribution, identified by two interviewees, is that of antimicrobial stewardship. Although this occurs after the drug development process and is therefore outside the scope of this paper, it is worth noting that altering prescribing practice involves changing the behaviour of both clinicians and patients. Designing effective interventions to achieve this is likely to require the involvement of both parties.

Many of these activities described above were carried out face-to-face, in workshops or project meetings. Some activities, for example, amending information sheets, were often carried out via email.

\section{Value added}

There was a wide range of views among our interviewees on the value of PPI in antimicrobial research. In some cases, the contribution was seen as 'minimal' and at best contributing a 'subjective insight'. One interviewee saw public involvement as, 'a lot of the time pointless', and as only relevant for a 'fraction of the time' (interview 1).

However, another interviewee commented that, 'PPI is required at all stages (of a research project)' but that 'PPI has most impact at the planning stage' as it '...can be a really good informal check that there is clarity of purpose' (interview 4).

PPI was also seen as helpful in dealing with operational concerns as they 'crop up' (interview 2). One interviewee commented on how helpful public contributors can be in advising on recruitment strategies for research projects and ensuring the acceptability of research procedures and proposed interventions to research participants. For example, one research project involved the use of anal swabs. The public contributors were able to advise the researchers on how best to approach potential participants and discuss this issue with them in a way that minimised anxiety about the process, resulting in a significant boost to recruitment figures (interview 6).

There was evidence of acceptance, even among those more sceptical about the benefits of PPI in setting the direction of research.

'We should not do research because we as researchers think it is interesting to us and which patients think is never going to benefit them.' (interview 8)

\section{Main barriers to public involvement}

Some of the barriers to developing PPI in antimicrobial research, such as the lack of clearly identifiable patient groups to work with and the technical nature of some of the research, have already been commented on. Beyond this, it is clear from the interview data that we have collected that PPI is a new concept in the world of antimicrobial research. Several of our interviewees had only recently become aware of it as a concept and were unclear about what it meant or how to put it into practice. There was also scepticism about PPI's specific contribution to antimicrobial research.

For one interviewee, the main barrier to effective PPI is, 'Lack of knowledge and experience of the area'. He commented that from his experience, the impact of PPI had been variable and this was related to the variable quality of PPI practice and facilitation. He saw this as a result of the PPI field being 'relatively immature'. As he put it, 'we are all learning how to do it' (interview 4). These comments could apply to the general development of PPI in research but are particularly relevant to the area of antimicrobial research. It may be inferred from these comments that as skill and expertise are developed in carrying out PPI in antimicrobial research, the beneficial impacts will increase. This represents both a challenge and an opportunity for the PPI community.

\section{DISCUSSION}

Although very little has been published about PPI in antimicrobial research, our small study suggests that, at least in the UK context, significant PPI work is taking place; however, this work is rarely written up for publication. This experience, although mixed, suggests that despite some initial scepticism, many researchers have found PPI beneficial to their work. As one UK-based interviewee put it, 'Now that we do it I wouldn't be without it'.

The greater uptake of PPI in the UK may simply reflect the fact that many research funders have made evidence of PPI a prerequisite for a successful application, although this begs the question, why have many UK funders taken this stance in the first place? There has been some preliminary work done on different 'cultures of involvement' in different parts of Europe. ${ }^{13}$ This may be an issue that is worthy of further exploration and will need to be taken 
in to account if PPI is to be implemented successfully in different regions of Europe.

Most of the PPI activity described by our interviewees related to the design and running of clinical trials. The contribution that PPI could make to laboratory-based research was absent, although PPI in this area could play a significant role in helping researchers to develop transparency, accountability and communication of their work to the wider public. Evidence from other areas of basic research suggests that PPI can help in the development of research questions and outcome measures in laboratory-based research. ${ }^{14}$

One of our interviewees suggested that substantial training would be required before public contributors could be involved throughout the medicines development process. EUPATI provides this kind of training and sees it as essential to enabling patients to act as effective advocates. However, some writers warn that an unintended consequence of this training may be to create groups of patients who identify too closely with the concerns of researchers rather than providing an alternative patient perspective. ${ }^{15}$ In our work with PPIPAD, we have found that some training is necessary to enable constructive discussions to take place, for example, on the medicines development process, but we did not find that this undermined the ability of panel members to present their own view point.

Unease was also raised about the representativeness of potential public contributors. This is an issue which has been widely debated in the PPI literature.$^{16}$ It is important to keep in mind that what is required in PPI is not statistical representativeness but what may be termed 'experiential representativeness', that is, representation of people with the experiential knowledge that is most relevant to the work in hand. However, concerns that public contributors are drawn from a relatively narrow section of society seem well founded and are reflected in the wider PPI literature.

Some interviewees also seemed to view the potential benefits of PPI in relatively narrow terms, that is, solely related to experience of an infection which is transitory. Unlike public contributors with chronic conditions, they did not see public contributors in antimicrobial research as developing 'expertise' in their own illness. However, others saw the potential for public contributors to play a wider range of roles, including acting as 'research ambassadors' and helping to create a more research receptive public.

Given the potential time and energy required to locate and involve appropriate public contributors in this area of work, the lack of clarity of the potential benefits of PPI and doubt about the ability of the public to engage with the issues, it is perhaps not surprising that many researchers in this area appear not to prioritise PPI in their work.

However, although individuals expressed scepticism about the contribution that PPI could make to different stages of the medicines development life cycle, collectively our interviewees identified a range of potential benefits of PPI covering most stages of the medicines development process. Due to the lack of published work in this area, there has been little opportunity for the researchers leading PPI to share and learn from each other's experiences.

\section{Strengths and limitations}

Our interview sample is small and recruited via personal contacts. It is in no way representative of researchers in the antimicrobial research community. However, our aim was not to map PPI activity in antimicrobial research, but to ascertain what approaches to PPI in antimicrobial medicines development are currently being used, the impacts that these approaches are having, and barriers to implementing these approaches-this, we were able to do. While it is possible that our sample is biased and represents a partial view of the issues discussed, it is unlikely that the issues are unique to our interviewees. In fact, many of the issues raised are recognisable in the wider PPI literature. ${ }^{78}$

We chose to undertake the interviews via telephone. It is possible that the lack of body language cues and personal interaction may have had an effect on the quality of the data collected. The data we collected were not of a personal or sensitive nature so this may have had less of an impact than in some other areas of research. ${ }^{17}$

\section{Concluding comments}

A major issue in developing PPI in antimicrobial medicines development research will be in overcoming the view that, at best, PPI has only a marginal contribution to make in this area of research. The findings from this study, although mixed, suggest that well-designed PPI has an untapped potential to enhance antimicrobial research. The difficulty is in breaking the cycle of low expectations, leading to low investment, leading to low impact and so on. ${ }^{18}$ In the UK, this cycle has begun to break down. This has been brought about by, among other things, research funders making PPI a mandatory part of grant applications. It may be that similar measures will need to be adopted in Europe and elsewhere to break this cycle, although the possibility that different attitudes to involvement may exist in different parts of Europe may also need to be explored and taken into account. However, it is clear that significant knowledge about the benefits of PPI in antimicrobial research is already beginning to be accumulated. Unfortunately, this practice-based knowledge is invisible to the wider academic community because it has not been published.

An important prerequisite for the future development of PPI in antimicrobial research will be the provision of clear and easily accessible guidance to researchers in this field on how to conduct PPI and the evidence of its benefits. Organisations like EUPATI have already made great strides in this direction. In order to tackle the issues raised in this article, the authors have also developed a 
toolkit for PPI in antimicrobial medicines development research. ${ }^{19}$

Importantly, none of our interviewees expressed hostility to the concept of PPI but several remained to be convinced of its value. Reassuringly, it appears that the researchers with direct experience of PPI were also the most positive about its benefits. With this in mind, we leave the final word to one of our interviewees:

'Go in to it (PPI) with an open mind and be prepared to be surprised about how valuable it will be.'

Acknowledgements We would like to thank the members of Patient and Public Involvement Panel for Antimicrobial Drugs (PPIPAD) for their help and support. We also acknowledge Julie Gibbs (Research Administrator for Microbiology, NBT) for her invaluable practical input into supporting the PPIPAD. The research leading to these results was conducted as part of the COMBACTE-MAGNET consortium. For further information please refer to www.COMBACTE.com.

Contributors $A G, D E, S G$ and $A M$ were involved in developing the concept and design of the study. AG and MK collected the data. AG analysed the data. All authors were involved in drafting and revising the manuscript. All authors read and approved the final manuscript.

Funding This research project receives support from the Innovative Medicines Initiative Joint Undertaking under grant agreement no. 115523 | 115620 | 115737 resources of which are composed of financial contribution from the European Union Seventh Framework Programme (FP7/2007-2013) and EFPIA companies in kind contribution.

Competing interests None declared.

Patient consent for publication Not required.

Ethics approval Ethical approval to carry out this study was obtained from the Faculty of Health and Applied Sciences Ethics Committee (FREC) at the University of the West of England. Informed consent was obtained before interviews commenced.

Provenance and peer review Not commissioned; externally peer reviewed.

Open access This is an open access article distributed in accordance with the Creative Commons Attribution Non Commercial (CC BY-NC 4.0) license, which permits others to distribute, remix, adapt, build upon this work non-commercially, and license their derivative works on different terms, provided the original work is properly cited, appropriate credit is given, any changes made indicated, and the use is non-commercial. See: http:// creativecommons.org/licenses/by-nc/4.0/.

\section{REFERENCES}

1. NIHR INVOLVE. What is public involvement in research? http://www. invo.org.uk/find-out-more/what-is-public-involvement-in-research-2/ (Accessed 29 Nov 2017).

2. Gibson A, Britten N, Lynch J. Theoretical directions for an emancipatory concept of patient and public involvement. Health 2012;16:531-47.

3. Armstrong N, Herbert G, Aveling EL, et al. Optimizing patient involvement in quality improvement. Health Expect 2013;16:e36-47.

4. Brown LJE, Dickinson T, Smith S, et al. Openness, inclusion and transparency in the practice of public involvement in research: a reflective exercise to develop best practice recommendations. Health Expectations 2018;21:441-7.

5. Evans D, Bird E, Gibson A, et al. Extent, quality and impact of patient and public involvement in antimicrobial drug development research: A systematic review. Health Expect 2018;21:75-81.

6. O'Neill J. Review on antimicrobial resistance. London: Department of Health, 2016.

7. Staley K. Exploring impact: public involvement in NHS, Public Health and Social Care Research. Eastleigh: INVOLVE, 2009.

8. Brett J, Staniszewska S, Mockford C, et al. Mapping the impact of patient and public involvement on health and social care research: a systematic review. Health Expect 2014;17:637.50.

9. Epstein S. The construction of lay expertise: AIDS activism and the forging of credibility in the reform of clinical trials. Sci Technol Human Values 1995;20:408-37.

10. Silverman D. Doing qualitative research a practical handbook. 5th edn. London: Sage, 2017.

11. Ritchie J, Spencer L. Qualitative data analysis for applied policy research by Jane Ritchie and Liz Spencer in A. Bryman and R. G. Burgess [eds.] 'Analysing qualitative data. London: Routledge, 1994:173-94.

12. Gibson A, Welsman J, Britten N. Evaluating patient and public involvement in health research: from theoretical model to practical workshop. Health Expect 2017;20:826-35.

13. Dent M, Pahor M. Patient involvement in Europe--a comparative framework. J Health Organ Manag 2015;29:546-55.

14. INVOLVE. NIHR Senior Investigators: leaders for patient and public involvement in research. Eastleigh: INVOLVE, 2014.

15. Ives J, Damery S, Redwod S. PPI, paradoxes and Plato: who's sailing the ship? J Med Ethics 2013;39:181-5.

16. Martin GP. 'Ordinary people only': knowledge, representativeness, and the publics of public participation in healthcare. Sociol Health IIIn 2008;30:35-54.

17. Sturges JE, Hanrahan KJ. Comparing telephone and face-to-face qualitative interviewing: a research note. Qualitative Research 2004;4:107-18.

18. Snape D, Kirkham J, Preston J, et al. Exploring areas of consensus and conflict around values underpinning public involvement in health and social care research: a modified Delphi study.(2014). BMJ Open 2014:6:1-10.

19. Kok M, Gibson A, Evans D, et al. Practical guide: patient and public involvement in antimicrobial medicines development research. Manual. Bristol: University of the West of England, 2018. 